\title{
Supporting Information: Polariton Transitions in Femtosecond Transient Absorption Studies of Ultrastrong Light-molecule Coupling
}

Courtney A. DelPo ${ }^{\dagger}$, Bryan Kudisch ${ }^{\dagger, \perp}$, Kyu Hyung Park ${ }^{\dagger, \perp}$, Saeed-Uz-Zaman Khan ${ }^{\ddagger}$, Francesca

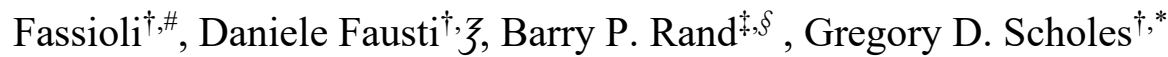

$\dagger$ Department of Chemistry, Princeton University, Princeton, New Jersey 08544, United States $\ddagger$ Department of Electrical Engineering, Princeton University, Princeton, New Jersey 08544, United States

$\S$ Andlinger Center for Energy and the Environment, Princeton University, Princeton, New Jersey 08544, United States

\#SISSA - Scuola Internazionale Superiore di Studi Avanzati, Trieste 34136, Italy

3Department of Physics, University of Trieste, Via A. Valerio 2, 34127 Trieste, Italy ZElettra-Sincrotrone Trieste S.C.p.A. Strada Statale 14 - km 163.5 in AREA Science Park 34149 Basovizza, Trieste, Italy

*Email: gscholes@princeton.edu

${ }^{\perp}$ These authors contributed equally.

Table of Contents

Experimental Section

Supplementary Figure 1: Bare film characterization

Supplementary Figure 2: Q-factor calculation

Supplementary Figure 3: TE and TM polarized PL spectra

Supplementary Figure 4: Spectroelectrochemistry

Supplementary Figure 5: Power dependence

Supplementary Figure 6: Detuned cavity transient spectra

Supplementary Figure 7: Off-resonant excitation of cavity

Supplementary Figure 8: Enhancement of UP GSB when pumping LP

Supplementary Figure 9: Global analysis pumping UP

Supplementary Figure 10: Global analysis pumping LP

Supplementary Figure 11: UP to DS transition and theoretical calculation

Theory Section 


\section{Experimental Section:}

Cavity fabrication: 4CzIPN (Ossila) was used as received. Bathocuproine (BCP) (Lumtec) was purified via thermal gradient sublimation prior to use. Quartz (AdValue Technology) substrates were cleaned by successive sonication in deionized water $\left(40^{\circ} \mathrm{C}, 5 \mathrm{~min}\right)$, acetone $\left(40{ }^{\circ} \mathrm{C}, 5 \mathrm{~min}\right)$ and isopropanol $\left(40^{\circ} \mathrm{C}, 5 \mathrm{~min}\right.$ ), followed by oxygen plasma treatment for $5 \mathrm{~min}$. To create the cavity, $30 \mathrm{~nm}$ thick layer of Ag (Kurt J. Lesker) was deposited on quartz followed by coevaporation of 4CzIPN and BCP (10:1 volume ratio) and another $200 \mathrm{~nm}$ top layer of Ag. Thermal evaporation was done inside (Angstrom EVOVAC Thermal Evaporation System) with $\sim 10^{-7}$ torr base pressure. Thickness of the 4CzIPN:BCP layer was varied from 151-159 nm. Profilometry was performed using a KLA Tencor P-15 Profilometer to confirm the actual thickness of the organic layer in the cavity was approximately $130 \mathrm{~nm}$.

Steady-state measurements: Angle-dependent reflection experiments were performed on a UVVis Cary 5000 Spectrometer with the Universal Measurement Accessory attached. Angledependent fluorescence was performed on a home-built setup. A $406 \mathrm{~nm}$ excitation laser (Thorlabs CPS405) focused to the sample at normal incidence and photoluminescence was collected by changing the angle of the detector arm mounted on the rotating breadboard (Thorlabs, RBB12A). Collected photoluminescence was fed into the optical fiber connected to a portable spectrometer (Ocean Optics, USB4000). Angle-resolution of the measurement was $6^{\circ}$.

Transient reflection experiments: Description of the pump-probe setup is provided elsewhere ${ }^{1}$. Angle-dependent measurements were obtained by changing the position of the sample. An additional mirror was placed next to the sample to direct the reflected signal to the detector. 

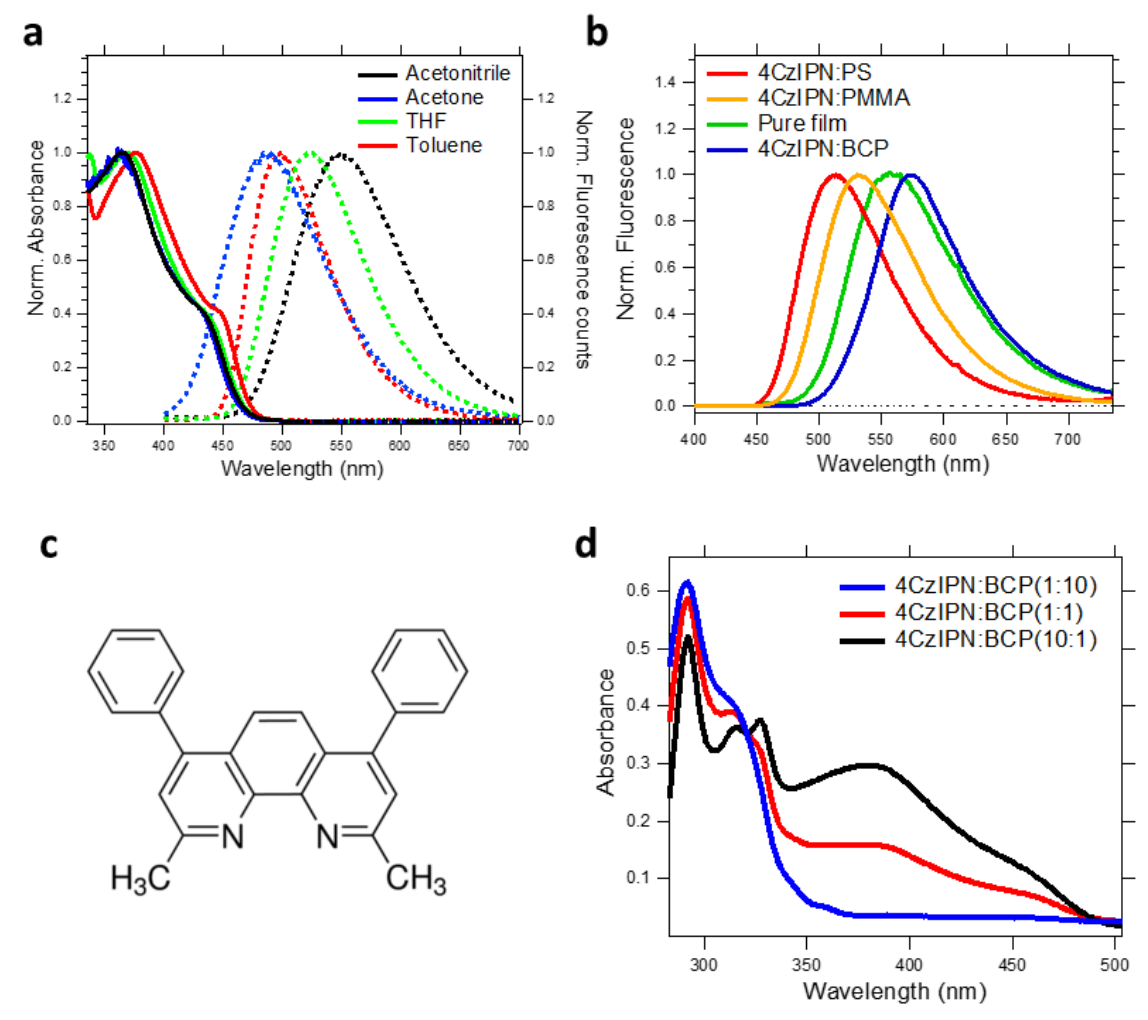

Supplementary Figure 1. Solvent (a) and medium (b) dependence of the steady-state properties of 4CzIPN. (c) Structure of BCP (bathocuproine). (d) Contribution of BCP absorption to 4CzIPN absorption at varying concentrations.

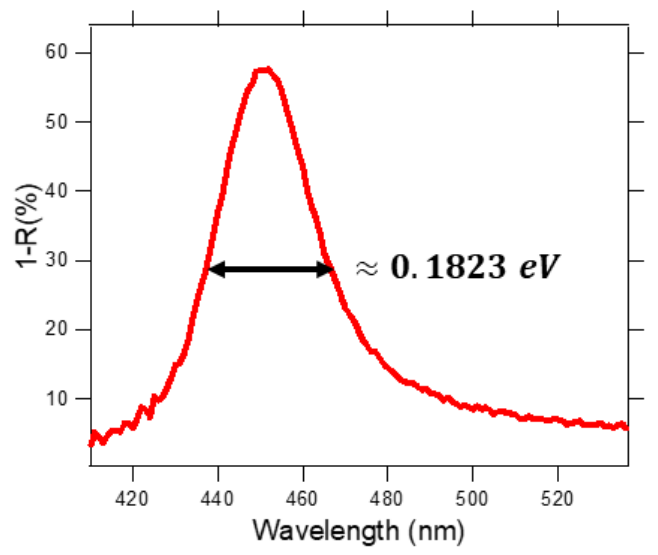

Supplementary Figure 2. Determination of Q-factor. Reflection spectrum of a bare cavity of thickness $130 \mathrm{~nm}$. The FWHM of the peak is approximately $0.1823 \mathrm{eV}$. The energy of the transition is approximately $2.725 \mathrm{eV} \cdot Q=\frac{2.725 \mathrm{eV}}{0.1823 \mathrm{eV}} \approx 15$. 

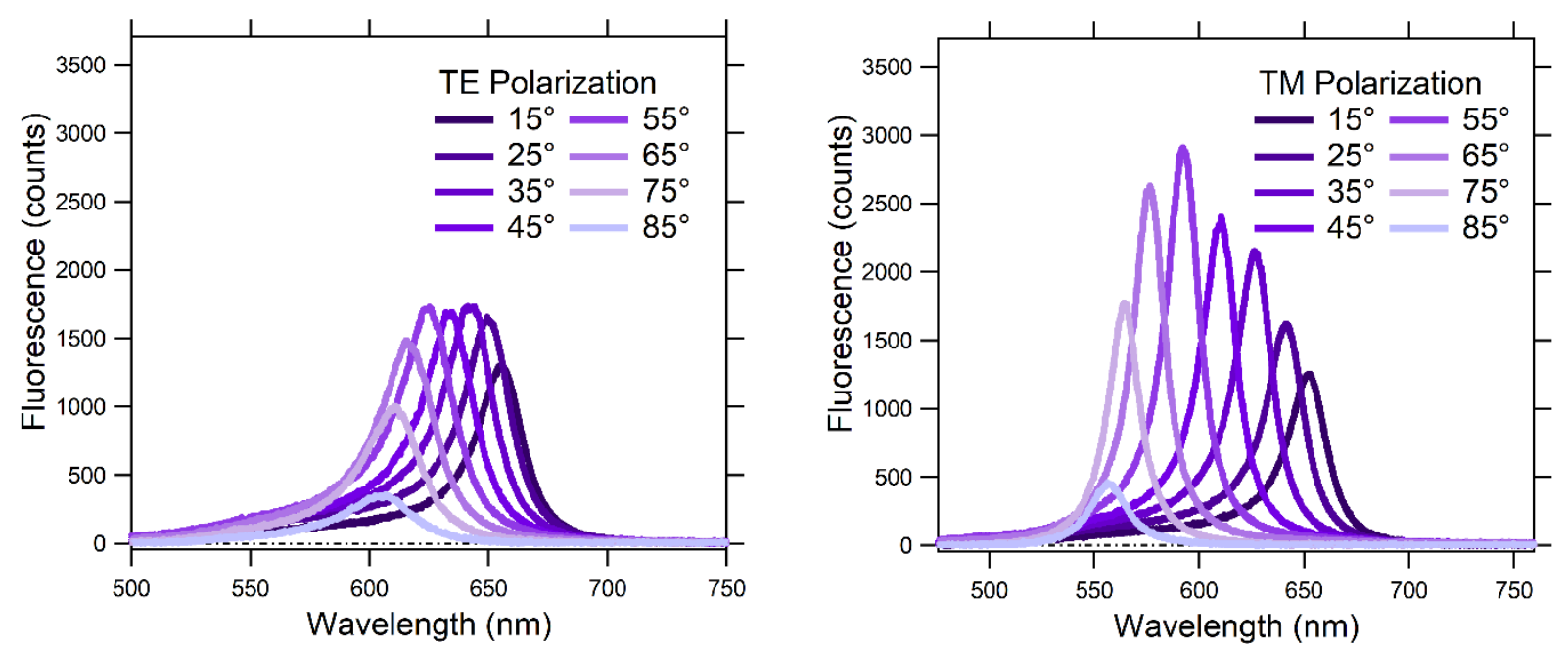

Supplementary Figure 3. Angle-dependent fluorescence spectra of the cavity excited at $406 \mathrm{~nm}$ with TE and TM polarization. A polarizer was placed before the detector to adjust the polarization of the fluorescence.

a

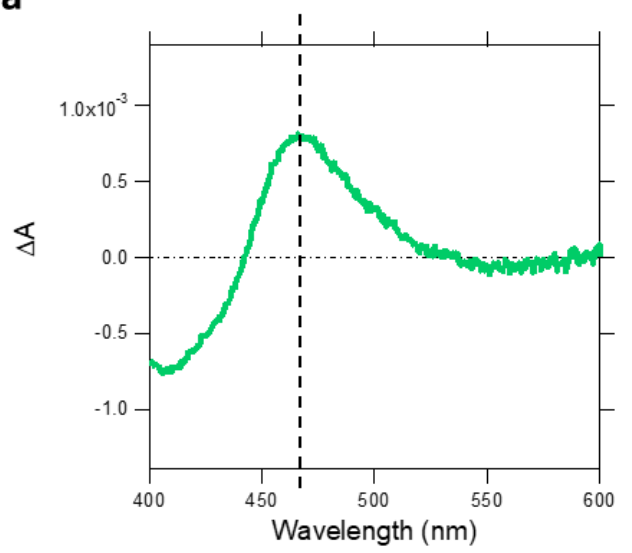

b

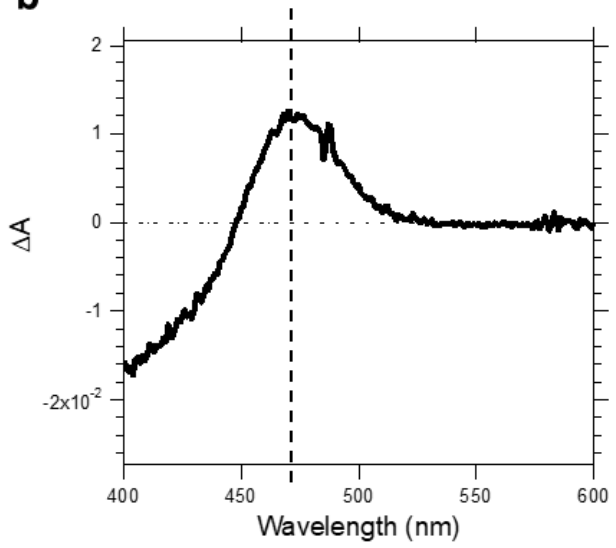

Supplementary Figure 4. Confirmation of spectral features of 4CzIPN outside of a cavity with spectroelectrochemistry. (a) Pump-probe spectrum at $7 \mathrm{~ns}$ of 4CzIPN in acetonitrile solution ex. $370 \mathrm{~nm}$ with pump power $130 \mathrm{uW}$. (b) Absorption of 4CzIPN in acetonitrile solution with the reducing agent tetrabutylammonium hexafluorophosphate at $0 \mathrm{~s}$ subtracted from spectrum at $120 \mathrm{~s}$ with the application of $1075 \mathrm{meV}$ to induce the reduction of the dicyanobenzene center. 


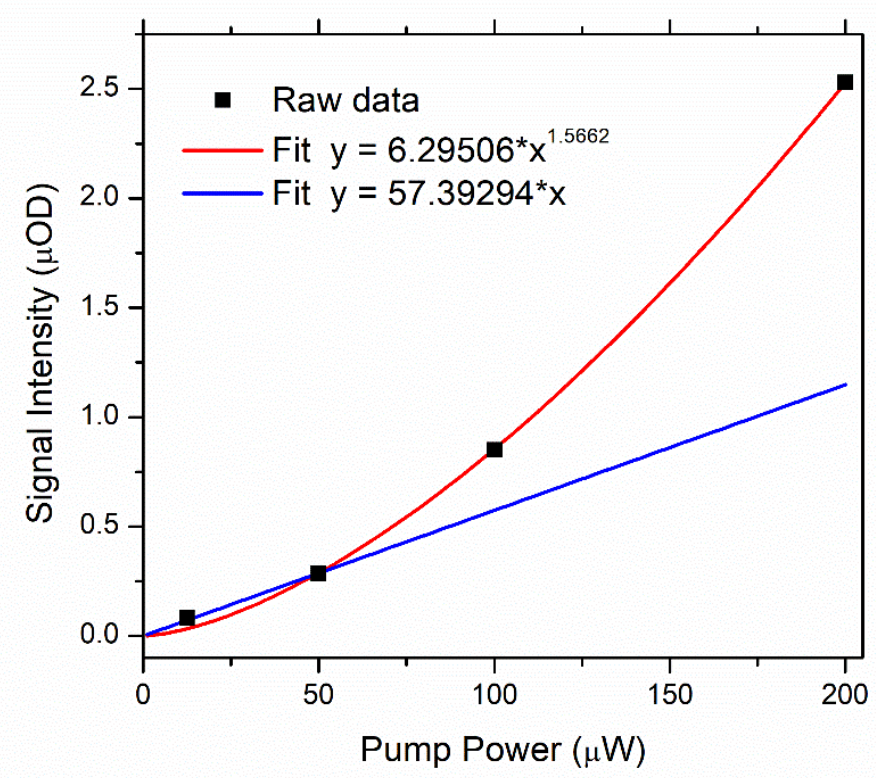

Supplementary Figure 5. Extended power dependence of the LP energy feature when pumping the LP at higher powers. Signal intensity recorded at $100 \mathrm{fs}$. Fit in solid red curve displays the superlinear dependence of the signal at high pump powers which is evidence for two-photon absorption. Pump-probe spectra in the main text was recorded at the linear power regime. 

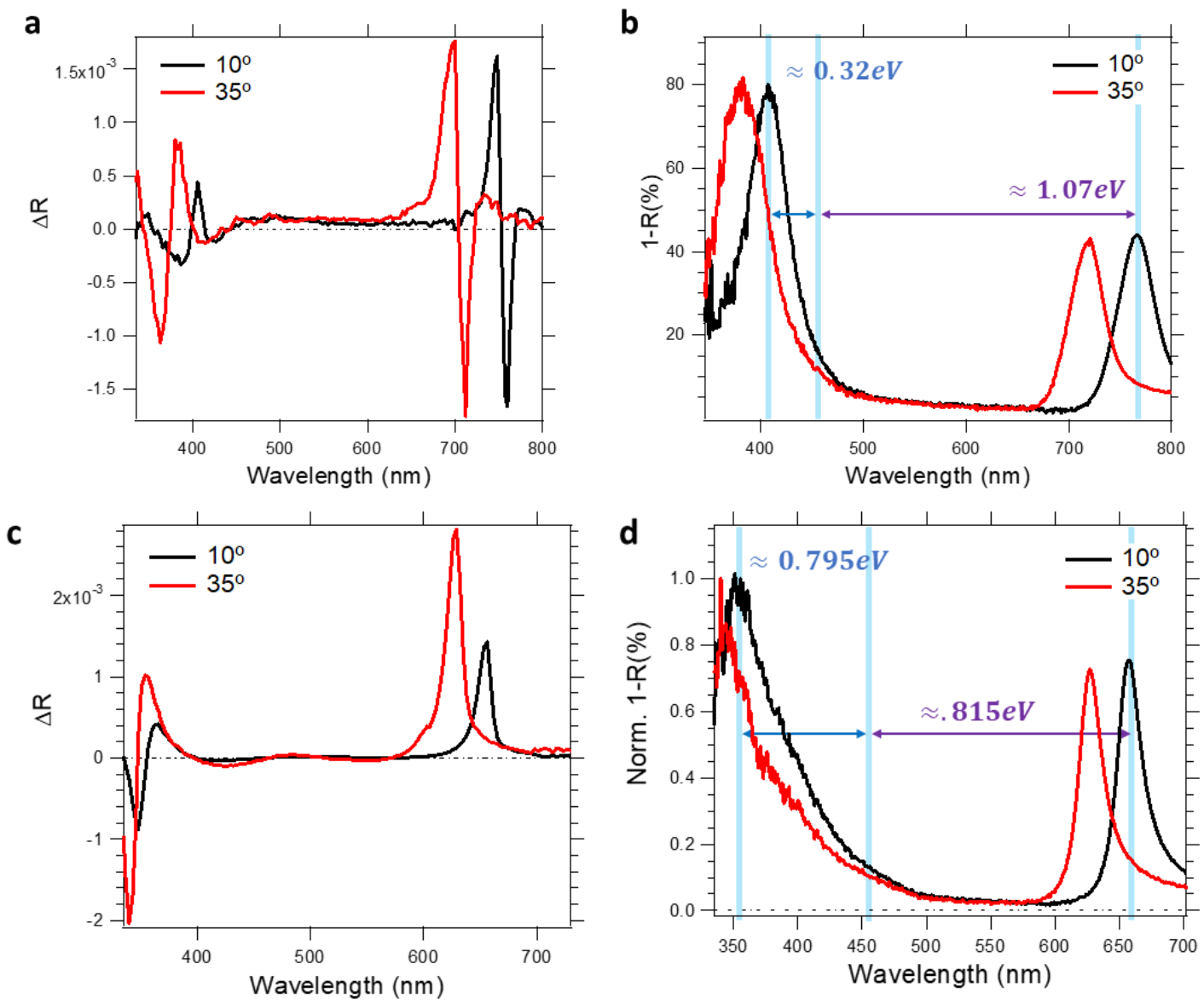

Supplementary Figure 6. Detuned cavity spectral features. Pump-probe spectra at 200 ps for an off resonant cavity pumped at $370 \mathrm{~nm}$ and angled at $10^{\circ}$ and $35^{\circ}$ (a) with accompanying reflection spectra (b). The off-resonant cavity possesses uneven Rabi splitting as indicated by the magnitude of splitting from the UP to the 4CzIPN singlet (blue) and the LP to the 4CzIPN singlet (purple). Due to the uneven splitting, the overlapping ESA features at the UP and LP energies are not observed. For comparison, the spectra produced when pumping a resonant cavity with approximately even Rabi splitting are displayed in (c) and (d). 

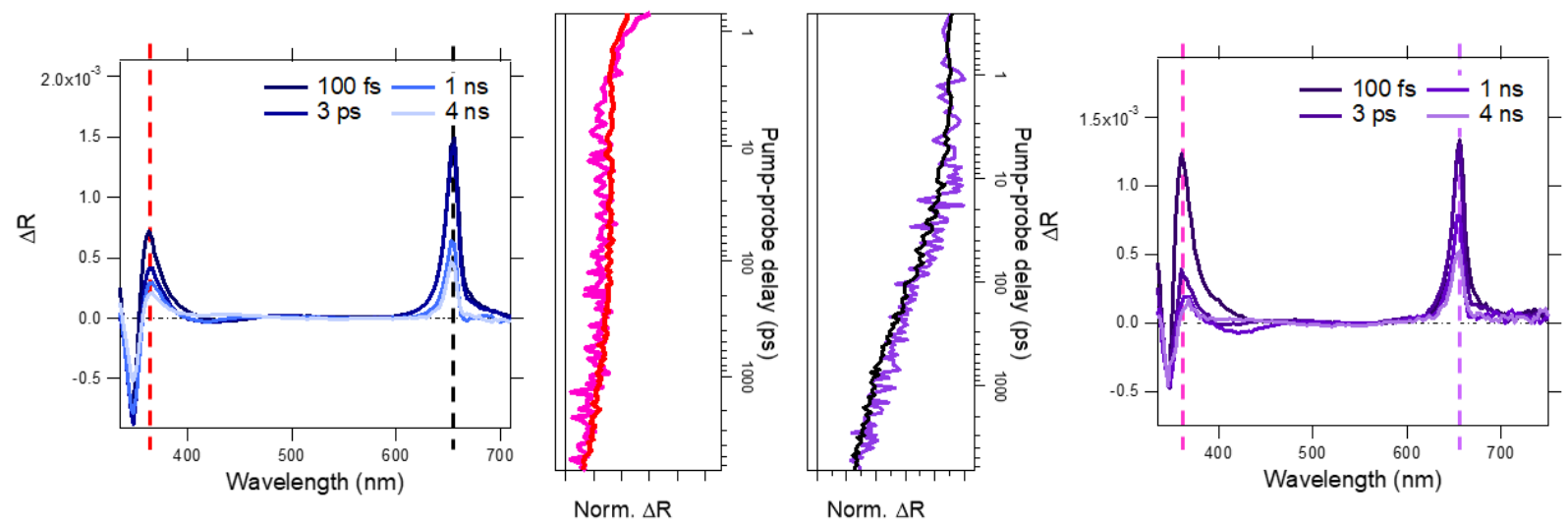

Supplementary Figure 7. Off-resonant excitation. Comparison of the dynamics when pumping the upper polariton at $370 \mathrm{~nm}$ (blue) to pumping at $450 \mathrm{~nm}$ (purple). Upper polariton energy feature dynamics are shown in red for pumping $370 \mathrm{~nm}$ and pink for pumping $450 \mathrm{~nm}$. Lower polariton energy feature dynamics are shown in black for pumping $370 \mathrm{~nm}$ and in purple for pumping $450 \mathrm{~nm}$. Pumping at $450 \mathrm{~nm}$ required a much higher pump power of $100 \mu \mathrm{W}$. The dynamics are the same at both pump wavelengths with the exception of the larger population transfer to the lower polariton when pumping at $450 \mathrm{~nm}$. Similar population transfer effect as pumping at an angle.
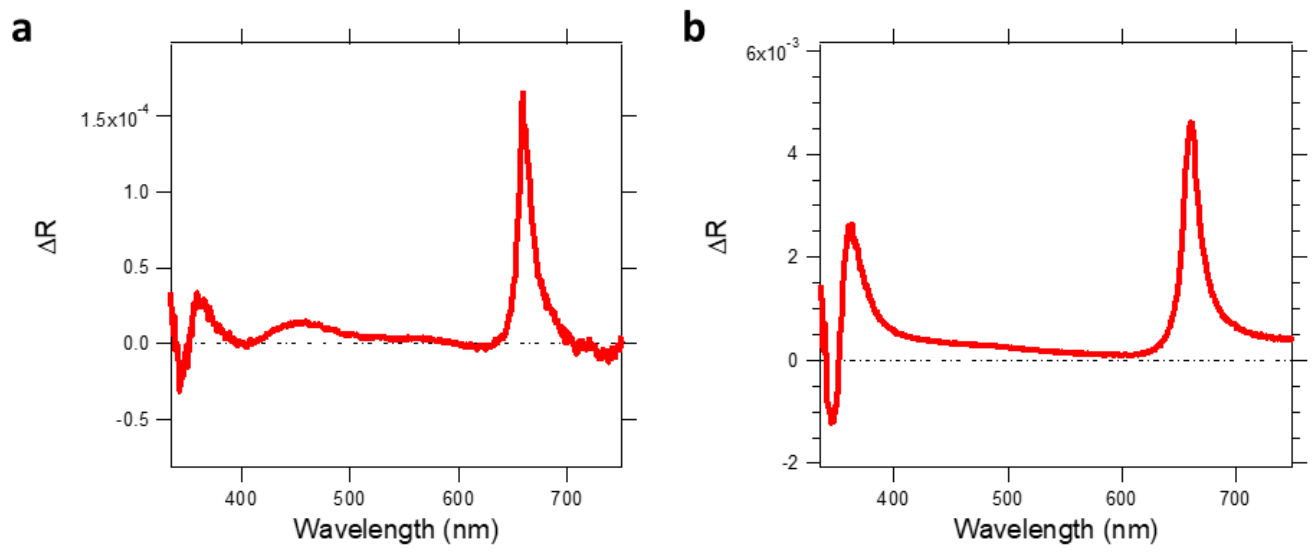

Supplementary Figure 8. Pump-probe spectrum averaged over all time points to observe the upper polariton derivative-like bleach feature when pumping the lower polariton at $12.5 \mu \mathrm{W}$ (a) and $200 \mu \mathrm{W}$ (b). Spectrum in (b) is shown because of the reduced noise. 

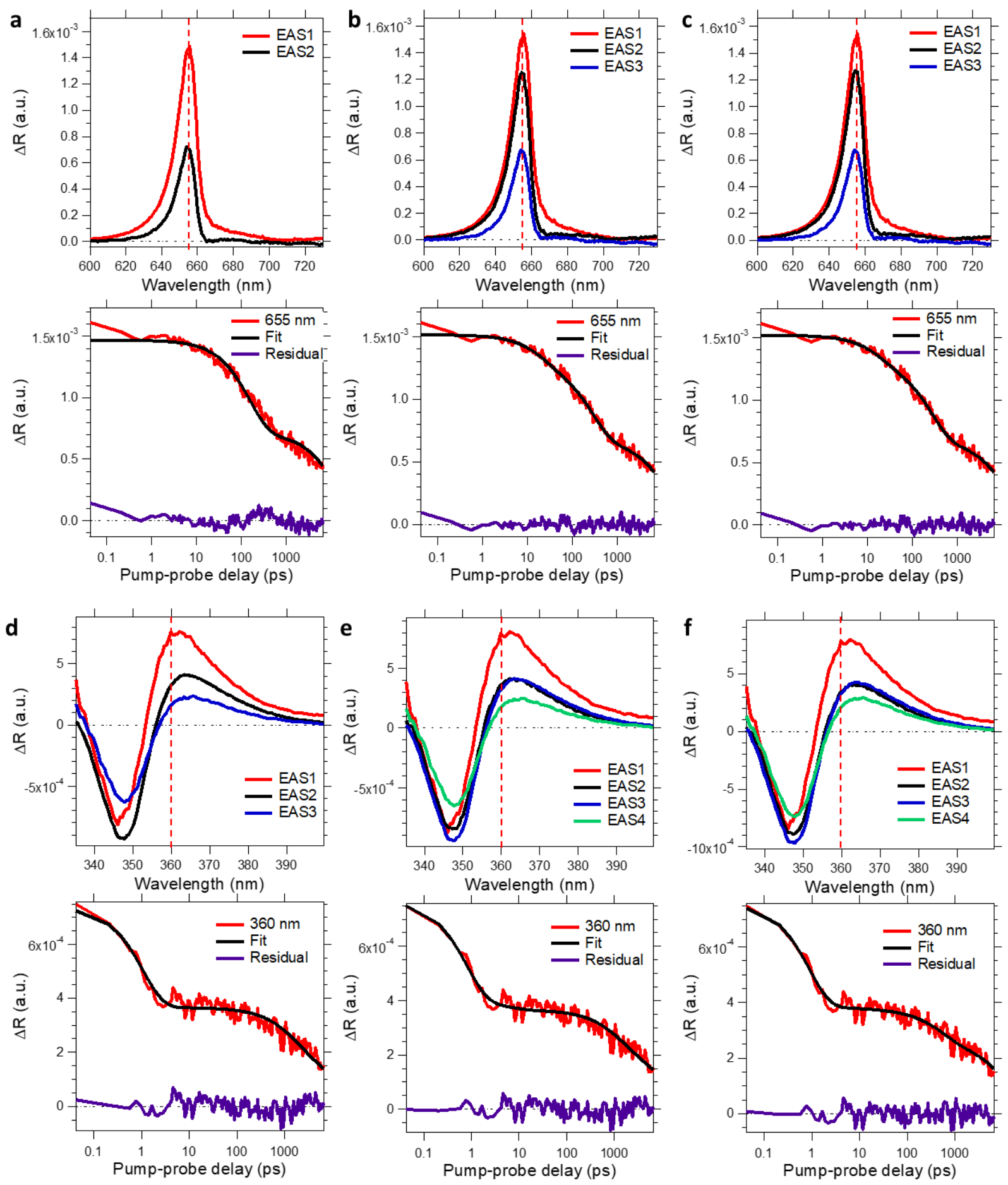


\begin{tabular}{r|llll}
\multicolumn{1}{c}{$\boldsymbol{\tau}_{\mathbf{1}}$} & \multicolumn{1}{c}{$\boldsymbol{\tau}_{\mathbf{2}}$} & \multicolumn{1}{c}{$\boldsymbol{\tau}_{\mathbf{3}}$} & \multicolumn{1}{c}{$\boldsymbol{\tau}_{\mathbf{4}}$} \\
\hline $\boldsymbol{a}$ & $153.0 \mathrm{ps}$ & $13.91 \mathrm{~ns}^{*}$ & - & - \\
$\boldsymbol{b}$ & $22.20 \mathrm{ps}$ & $284.2 \mathrm{ps}$ & $13.91 \mathrm{~ns}^{*}$ & - \\
$\boldsymbol{c}$ & $19.42 \mathrm{ps}^{*}$ & $276.9 \mathrm{ps}$ & $13.91 \mathrm{~ns}^{*}$ & - \\
$\boldsymbol{d}$ & $1.075 \mathrm{ps}$ & $1.532 \mathrm{~ns}$ & $13.91 \mathrm{~ns}^{*}$ & - \\
$\boldsymbol{e}$ & $788.1 \mathrm{fs}$ & $5.849 \mathrm{ps}$ & $1.256 \mathrm{~ns}^{*}$ & $13.91 \mathrm{~ns}^{*}$ \\
$\boldsymbol{f}$ & $888.3 \mathrm{fs}$ & $19.42 \mathrm{ps}^{*}$ & $529.1 \mathrm{ps}^{*}$ & $13.91 \mathrm{~ns}^{*}$ \\
*Time constants fixed to 4CZIPN parameters & &
\end{tabular}

Supplementary Figure 9. Global analysis results for the data pumping the upper polariton (370 nm) and probing the $660 \mathrm{~nm}$ feature (a, b, c) and the $360 \mathrm{~nm}$ feature (d, e, f). The final time constant for all features was fixed to the final time constant for 4CzIPN, $13.914 \mathrm{~ns}$. For the $660 \mathrm{~nm}$ feature, 3 states fit best and for the $360 \mathrm{~nm}$ feature 4 states fit best as evidenced by the residual improvement in b and c compared to a and in e and $\mathrm{f}$ compared to $\mathrm{d}$. . Fixing a larger number of time constants to 4CzIPN time constants (c, f) provides a similar result to free parameters $(b, e)$. 

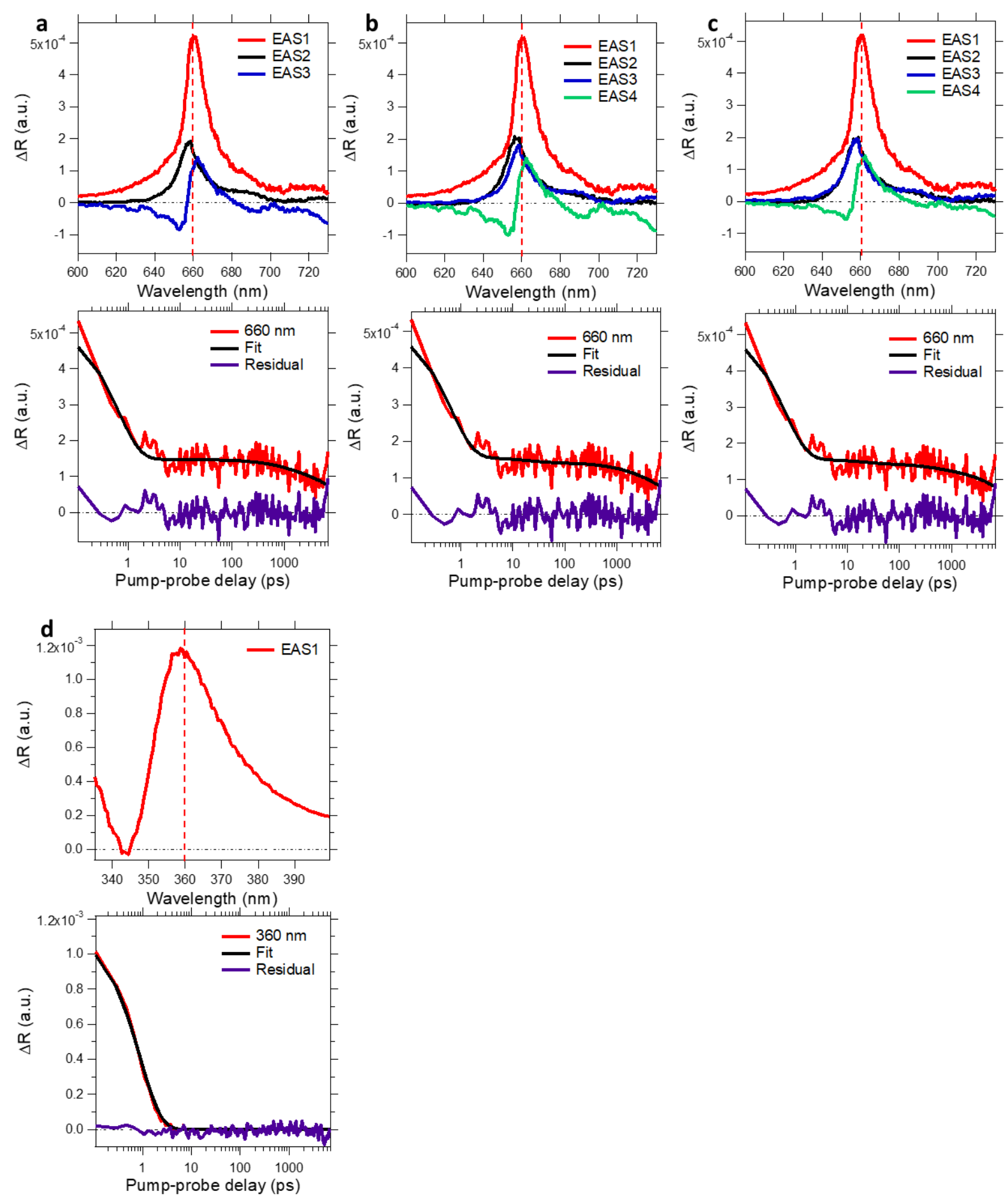


\begin{tabular}{r|rlll} 
& $\boldsymbol{\tau}_{\mathbf{1}}$ & $\boldsymbol{\tau}_{\mathbf{2}}$ & \multicolumn{1}{c}{$\boldsymbol{\tau}_{\mathbf{3}}$} & \multicolumn{1}{c}{$\boldsymbol{\tau}_{\mathbf{4}}$} \\
\hline $\boldsymbol{a}$ & $639.5 \mathrm{fs}$ & $1.111 \mathrm{~ns}$ & $13.91 \mathrm{~ns}^{*}$ & - \\
$\boldsymbol{b}$ & $645.8 \mathrm{fs}$ & $19.42 \mathrm{ps}^{*}$ & $1.714 \mathrm{~ns}^{*}$ & $13.91 \mathrm{~ns}^{*}$ \\
$\boldsymbol{c}$ & $622.2 \mathrm{fs}$ & $19.42 \mathrm{ps}^{*}$ & $529.1 \mathrm{ps}^{*}$ & $13.91 \mathrm{~ns}^{*}$ \\
$\boldsymbol{d}$ & $881.0 \mathrm{fs}$ & - & - & -
\end{tabular}

*Time constants fixed to 4CzIPN parameters

Supplementary Figure 10. Global analysis results for the data pumping the lower polariton (660 nm) and probing the $660 \mathrm{~nm}$ feature (a, b, c) and the $360 \mathrm{~nm}$ feature (d). The final time constant for all features was fixed to the final time constant for 4CzIPN, $13.914 \mathrm{~ns}$. For the $660 \mathrm{~nm}$ feature, 4 states fit best as evidenced by the residual improvement in $\mathrm{b}$ and c compared to a. Fixing a larger number of time constants to 4CzIPN time constants (c) provides a similar result to free parameters (b). For the $360 \mathrm{~nm}$ feature, 1 state fits best and the time constant cannot be fixed.

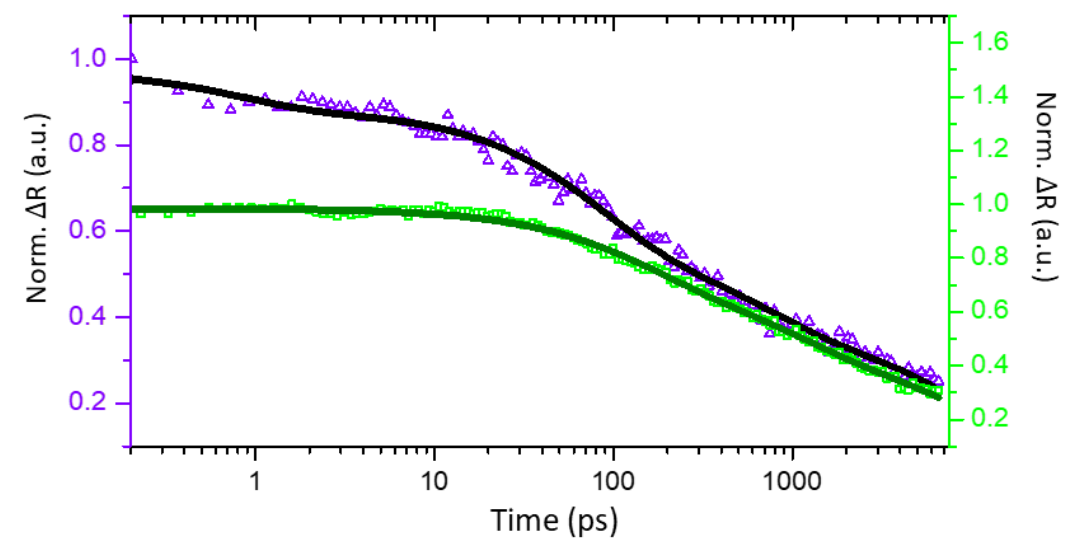

Supplementary Figure 11. Kinetic traces from the bare film ESA at $485 \mathrm{~nm}$ (green) and the ESA at 660 $\mathrm{nm}$ when pumping the UP (purple) replotted to highlight the existence of additionally fast decaying components within the tens of picosecond timescale in the cavity system when pumping the UP.

The observed lifetime of the UP and LP in J-aggregate microcavity systems is much faster than what we observe in the 4CzIPN system. The femtosecond scale lifetime in the J-aggregate systems has also been predicted using the equation below (reproduced from Ref. 2) ${ }^{2}$. Using the same equation with our experimental parameters, we calculate a predicted lifetime of $1.9 \mathrm{ps}$ which is significantly larger than that predicted and observed in the J-aggregate cavity systems.

$$
\begin{aligned}
W^{(\text {incoh })}(\boldsymbol{q})= & \frac{2 \pi g^{2} E_{\mathrm{vib}}^{2}}{\hbar \sqrt{\pi} \gamma_{0}} e^{-\left[E_{0}-\left(E_{\mathrm{U}}(\boldsymbol{q})-E_{\mathrm{vib}}\right)\right]^{2} / \gamma_{0}^{2}} \frac{\Delta^{2}}{\left[E_{0}-E_{\mathrm{U}}(\boldsymbol{q})\right]^{2}+\Delta^{2}} \\
& \tau^{\text {(incoh) }}(\boldsymbol{q})=\frac{1}{W^{(\text {incoh })(\boldsymbol{q})}}
\end{aligned}
$$

$E_{0}$ and $E_{\mathrm{U}}$ are transition energies of a bare molecule and of the UP, $\gamma_{0}$ is the broadening of excitonic resonance, $\Delta$ is a half of the Rabi splitting, $g$ is the coupling constant, and $E_{\text {vib }}$ is the energy of the vibrational mode. We used the following parameters obtained from our experiments: $E_{0}=2.74 \mathrm{eV}, E_{\mathrm{U}}=$ $3.54 \mathrm{eV}, \gamma_{0}=.28 \mathrm{eV}, \Delta=0.80 \mathrm{eV}$ and assumed $E_{\mathrm{vib}}=0.18 \mathrm{eV}$, an energy that corresponds to the $\mathrm{C}=\mathrm{C}$ stretching mode prevalent in organic molecules. The calculated time constant for our 4CzIPN cavity system, $\tau^{(\text {incoh })}$, is $1.9 \mathrm{ps,} \mathrm{which} \mathrm{is} \mathrm{drastically} \mathrm{larger} \mathrm{than} \mathrm{the} 70 \mathrm{fs}$ time constant calculated in the case of J-aggregate microcavities. This supports the fact that we see a kinetic difference shown in Supplementary 
Figure 11 above. We think that the difference lasting up to $100 \mathrm{ps}$ might be from a slower UP-to-DS transition, which is not captured by equation 31 assuming a simple Fermi's-golden-rule-type transition. We found that an important factor in controlling the transition rate is the energy gap between UP and DS, which is associated with the exponential term in eq (1).

\section{Theory}

In this section we present the energy structure of a cavity-coupled system predicted by the Tavis-Cummings model (Dicke model within the rotating wave approximation) that treats a collection of $\mathrm{N}$ equal two-level systems coupled to a single optical mode. Each two-level system is representative of the ground and relevant electronically excited molecular state that couples to the cavity mode. We provide the analytical solutions for the 0-, 1- and 2-particle states, which we relate in the main text to the optical transitions observed in the pump-probe experiments.

The Tavis-Cummings Hamiltonian is given by

$$
H=E \sum_{i=1}^{N} \sigma_{i}^{+} \sigma_{i}^{-}+\omega a^{\dagger} a+g \sum_{i=1}^{N}\left(\sigma_{i}^{+} a+\sigma_{i}^{-} a^{\dagger}\right)
$$

where $\sigma_{i}^{+(-)}$are the rising (lowering) operators of the `i-th' two level system with transition energy $E, a^{\dagger}(a)$ describe the creation (annihilation) operators of the bosonic optical field, $\omega$ is the frequency of the optical mode and $\mathrm{g}$ measures the strength of the interaction. Since the Hamiltonian Eq. 2 conserves the total number of particles $N_{p}=\sum_{i=1}^{N} \sigma_{i}^{+} \sigma_{i}^{-}+a^{\dagger} a$ (either molecular or photonic excitations), i.e. $\left[H, N_{p}\right]=0$, its eigenstates are a superposition of states with the same number of particles. In the following we will assume the case of resonance $(\omega=E)$ and focus on the solution of the eigenstates for the $N_{p}=1$ and $N_{p}=2$ particle cases. We also evaluate the molecular transition dipole moment $\tilde{\mu}=\mu_{m}\left(\sum_{i=1}^{N}\left|0_{i}\right\rangle\left\langle 1_{i}|+| 1_{i}\right\rangle\left\langle 0_{i}\right|\right)$ between the eigenstates of the light-matter Hamiltonian $\mathrm{H}$, where $\left|0_{i}(1)_{i}\right\rangle$ refer to the local ground (excited) state of the ' $i$-th' molecule and $\mu_{m}$ is the molecular transition dipole moment strength assumed to be independent of ' $i$ '.

The collective matter-photon states are labeled as $\left\{|G, n\rangle,\left|e_{i}, n\right\rangle\right.$ and $\left.\left|e_{i} e_{j}, n\right\rangle(i \neq j)\right\} . G, e_{i}$ and $e_{i} e_{j}$ label the molecular states and represent all molecules in the ground state, only a single molecule ' $i$ ' excited and two molecules ' $i$ ' and ' $j$ ' excited respectively. The $n$ instead labels the number of photons on each state.

\section{a. One-particle states}

There are N+1 single-particle states given by the lower polariton (LP), upper polariton (UP) and N-1 degenerate dark states at energy $\omega$.

The expression for UP and LP is given by

$$
|U P / L P\rangle=\frac{1}{\sqrt{2}}|G, 1\rangle \pm \frac{1}{\sqrt{2 N}} \sum_{i=1}^{N}\left|e_{i}, 0\right\rangle,
$$


with energies $E_{U P / L P}=\omega \pm g \sqrt{N}$. The (vacuum) Rabi splitting is defined as $\Omega_{R}=E_{U P}-E_{L P}=$ $2 g \sqrt{N}$.

UP and LP are optically bright from the ground state with a transition dipole moment strength given by

$|\langle U P|\tilde{\mu}| G, 0\rangle|=|\langle L P|\tilde{\mu}| G, 0\rangle|=,\mu \sqrt{\frac{N}{2}}$. The dark states at energy $\omega$ are purely molecular and take the form

$$
|\omega\rangle=\sum_{i=1}^{N} c_{i}\left|e_{i}, 0\right\rangle
$$

where the coefficients satisfy $\sum_{i=1}^{N} c_{i}=0$. As their name suggests, the dark states are not optically allowed from the ground state.

\section{b. Two-particle states}

The highest and lowest eigenstates of the light-matter Hamiltonian in the 2-particle subspace are given by the upper (2UP) and lower (2LP) polaritons

$$
|2 U P / 2 L P\rangle=\frac{1}{\sqrt{2}} \sqrt{\frac{N}{(2 N-1)}}|G, 2\rangle \pm \frac{1}{\sqrt{2 N}} \sum_{i=1}^{N}\left|e_{i}, 1\right\rangle+\frac{1}{\sqrt{N(2 N-1)}} \sum_{i=1, j>i}^{N}\left|e_{i} e_{j}, 0\right\rangle
$$

with energies $E_{2 U P / 2 L P}=2 \omega \pm 2 g \sqrt{N-0.5}$. Note that for finite $\mathrm{N}, E_{2 U P}<2 E_{U P}$ and $E_{2 L P}>2 E_{L P}$. In other words, the two-particle Rabi splitting $\Omega_{R}{ }^{(2)}=E_{2 U P}-E_{2 L P}$ is smaller than twice the vacuum Rabi splitting $\Omega_{R}$, i.e. $\Omega_{R}{ }^{(2)}<2 \Omega_{R}$. Only in the limit of infinite $\mathrm{N}, \Omega_{R}{ }^{(2)}=2 \Omega_{R}$.

The two-particle polariton 2UP(2LP) is optically allowed from the one-particle UP(LP) with a transition dipole moment strength given by $|\langle U P|\tilde{\mu}| 2 U P\rangle|=|\langle L P|\tilde{\mu}| 2 L P\rangle|=\mu\left(\frac{\sqrt{N}}{2}+\frac{(N-1)}{\sqrt{2(2 N-1)}}\right)$.

In addition to the 2UP and 2LP there is another polariton state in the two-particle manifold that is optically accessible from the LP and UP. This is a state located at twice the energy of the cavity mode $2 \omega$ :

$$
|2 \omega\rangle=\sqrt{\frac{N-1}{(2 N-1)}}|G, 2\rangle-\sqrt{\frac{2}{(N-1)(2 N-1)}} \sum_{i=1, j>i}^{N}\left|e_{i} e_{j}, 0\right\rangle
$$


The strength of the transition dipole moment from LP and UP is $|\langle U P|\tilde{\mu}| 2 \omega\rangle|=|\langle L P|\tilde{\mu}| 2 \omega\rangle|=$ $\mu \sqrt{\frac{2(N-1) N}{2 N-1}}$.

We note that the model also predicts a collection of degenerate molecular dark states at energy $2 \omega$. These are given by the (un-normalized) expression:

$$
|D 2 \omega\rangle \sum_{i=1, j>i}^{N} c_{i j}\left|e_{i} e_{j}, 0\right\rangle
$$

with coefficients that satisfy $\sum_{j \neq i} c_{i j}=0$. These states are optically forbidden from the 1-particle states.

Finally the model also predicts additional states between the 2LP and $2 \omega$ and $2 \omega$ and 2 UP states, which are given by the (un-normalized) expression

$$
|D 2 U P / D 2 L P\rangle=\sum_{i=1}^{N}{c^{\prime}}_{i}\left|e_{i}, 1\right\rangle \pm \sqrt{\frac{1}{N-2}} \sum_{i=1, j>i}^{N}\left(c^{\prime}{ }_{i}+c^{\prime}{ }_{j}\right)\left|e_{i} e_{j}, 0\right\rangle
$$

with coefficients that satisfy $\sum_{i=1}^{N} c^{\prime}{ }_{i}=0$. Energies are located at $E_{D 2 U P / D 2 L P}=2 \omega \pm g \sqrt{N-2}$. These states are optically dark from the LP and UP but optically allowed from the dark states $|\omega\rangle$ at energy $\omega$.

\section{References:}

(1) Pensack, R. D.; Ostroumov, E. E.; Tilley, A. J.; Mazza, S.; Grieco, C.; Thorley, K. J.; Asbury, J. B.; Seferos, D. S.; Anthony, J. E.; Scholes, G. D. Observation of Two TripletPair Intermediates in Singlet Exciton Fission. J. Phys. Chem. Lett. 2016, 7 (13), 2370-2375. https://doi.org/10.1021/acs.jpclett.6b00947.

(2) Agranovich, V. M.; Litinskaia, M.; Lidzey, D. G. Cavity Polaritons in Microcavities Containing Disordered Organic Semiconductors. Phys. Rev. B 2003, 67 (8), 085311. https://doi.org/10.1103/PhysRevB.67.085311. 